International Journal of Bifurcation and Chaos, Vol. 8, No. 8 (1998) 1733-1738

(c) World Scientific Publishing Company

\title{
DESYNCHRONIZATION TRANSITIONS IN RINGS OF COUPLED CHAOTIC OSCILLATORS
}

\author{
I. P. MARIÑO* and V. PÉREZ-MUÑUZURI \\ Group of Nonlinear Physics, Faculty of Physics, University of Santiago de Compostela, \\ 15706 Santiago de Compostela, Spain \\ M. A. MATÍAS ${ }^{\dagger}$ \\ Físca Teórica, Facultad de Ciencias, Universidad de Salamanca, 37008 Salamanca, Spain
}

Received July 31, 1997; Revised November 25, 1997

\begin{abstract}
Rings of chaotic oscillators coupled unidirectionally through driving are studied. While synchronization is observed for small sizes of the ring, beyond a certain critical size a desynchronizing transition occurs. In the two examples studied here the system exhibits a transition to periodic rotating waves for rings of Lorenz systems, while one finds a sort of chaotic rotating waves when Chua's circuit is used.
\end{abstract}

\section{Introduction}

Synchronization phenomena are pervasive in nature [Winfree, 1980; Strogatz \& Stewart, 1993] and, thus, many studies have been carried out, focusing particularly on limit-cycle oscillators. Less intuitive is probably the finding that chaotic systems may be made to get in synchrony [Fujisaka \& Yamada, 1983; Pecora \& Carroll, 1990], as chaos has been described as a situation in which a system gets out of synchronization with itself [Tang et al., 1982]. In the present work we shall use the synchronization method introduced in [Güémez \& Matías, 1995], that amounts to a generalization of the method introduced by Pecora and Carroll (PC). The idea is to avoid partitioning the response system in subsystems, introducing, instead, the driving signal at a particular place of the response system, i.e. without reducing the size of the latter. This property is particularly useful in the case that we are interested here: the design of arrays of coupled chaotic oscillators, as all the units in the array will be of the same type (will have the same dimension), without reducing the richness in possible dynamical behaviors of the system. This method has been used before and synchronization waves in linear arrays of chaotic oscillators have been obtained [Sánchez et al., 1997].

In the present work, we shall consider rings of coupled chaotic oscillators. These geometries may be relevant in a biological context, like in morphogenesis [Turing, 1952] or in the context of neural systems. Thus, for example, Central Pattern Generators (CPGs), i.e. assemblies of small number of neurons, capable of providing the necessary rhythm of muscular activity even in the absence of external stimuli. These CPGs are believed to play an important role in animal locomotion. In these CPGs the relevant points to be considered are the dynamics of the isolated neurons, e.g. periodic or chaotic, the interaction between the oscillators, and the way in which information is processed. An important aspect is that the resulting spatiotemporal patterns can be analyzed through symmetry arguments [Collins \& Stewart, 1994], and this

\footnotetext{
${ }^{*}$ E-mail: ines@fmmeteo.usc.es
}

${ }^{\dagger}$ E-mail: mam@sonia.usal.es 
allows one to study the different possible behaviors, stemming from symmetry-breaking bifurcations, and, thus, the transition between different animal gaits has been explained in this way by considering a model composed out of a ring of coupled oscillators [Collins \& Stewart, 1994]. Regarding the possibility that single neurons are chaotic, some evidences point in this direction [Hayashi \& Ishizuka, 1992].

In the present work we shall explore further the richness of dynamical behaviors that are possible in rings of unidirectionally coupled chaotic oscillators, considering as case examples the Lorenz and Chua systems. We shall perform our study by considering a reference state in which the behavior of the coupled systems is chaotic and uniform (synchronized), studying then the onset of instability, characterized in a Fourier representation by the instability in the $k=1$ mode. This will yield rotating waves that in one case are periodic while in the other chaotic.

\section{Rings of Lorenz Oscillators}

In this case we shall consider rings coupled in such a way that the dynamical behavior is defined by,

$$
\left.\begin{array}{rl}
\dot{x}_{j} & =\sigma\left(y_{j}-x_{j}\right) \\
\dot{y}_{j} & =R \bar{x}_{j}-y_{j}-x_{j} z_{j} \\
\dot{z}_{j} & =x_{j} y_{j}-b z_{j}
\end{array}\right\} \quad j=1, \ldots, N
$$

where the coupling enters through $\bar{x}_{j}$, that is defined as $\overline{x_{j}}=x_{j-1}$, with $\bar{x}_{1}=x_{N}$.

In this situation it was observed [Matías et al., 1997a] that the synchronized chaotic state is stable if the size of the ring is small enough, e.g. $N=2$ (see Fig. 1), while for a certain critical number, $N_{c}=3$ in the case of Lorenz model, an instability that destroys the uniform chaotic state occurs, leading to a rotating chaotic wave (see Fig. 2). We have performed studies in which the parameters of the system have been varied, with the result that the critical size, $N_{c}=3$ in all cases. Anyway, so far we have explored only the region in which all the oscillators are identical.

A noteworthy aspect of this desynchronization transition is that the time scale of the emerging rotating wave is, roughly, one order of magnitude faster than that of the uncoupled oscillators. This instability can be characterized by performing a linear stability of the small deviations around the synchronized state (see e.g. [Turing, 1952; Heagy

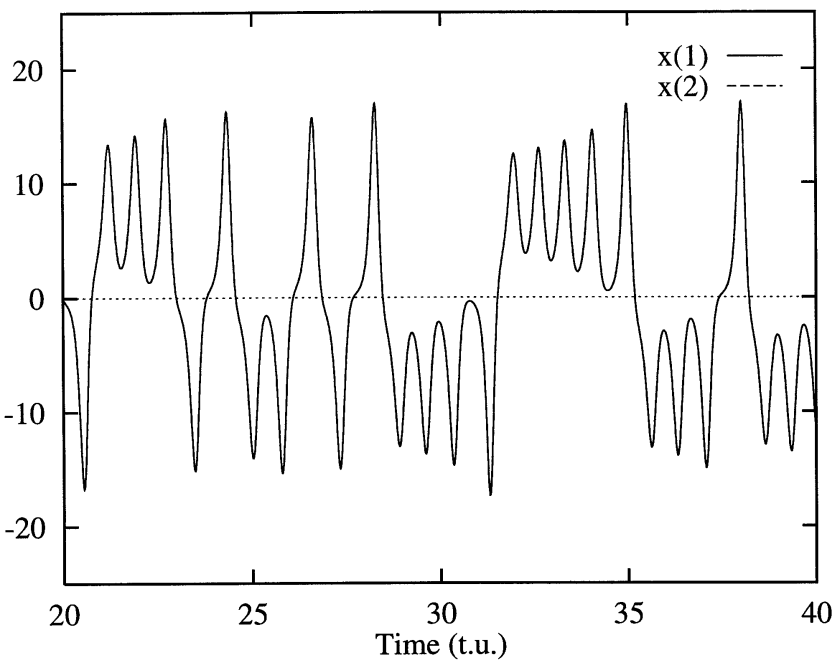

Fig. 1. Temporal evolution of the variable $x$ of the two oscillators in a ring of $N=2$ Lorenz oscillators. The values of the parameters are $(\sigma, R, b)=(10,28,8 / 3)$.

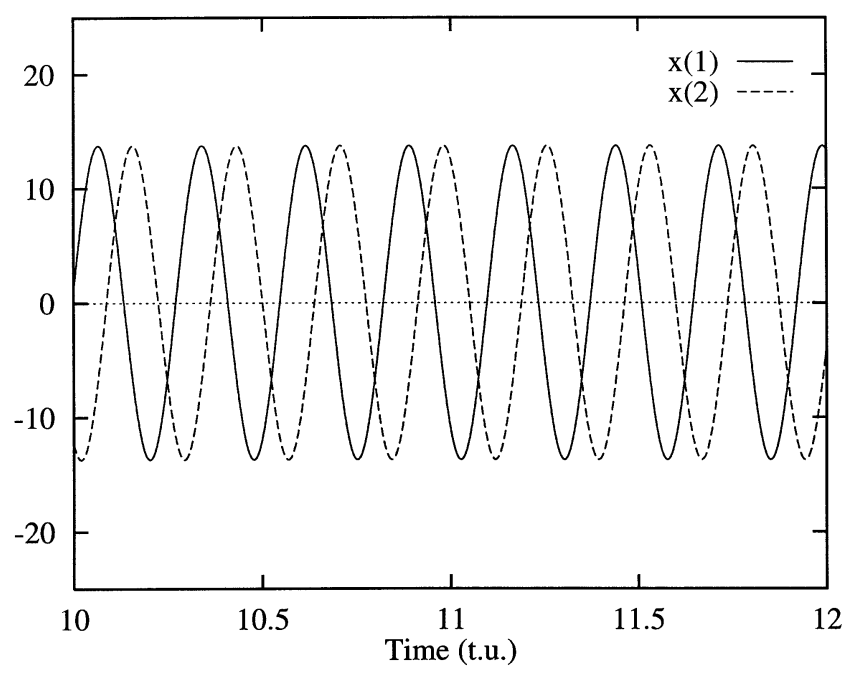

Fig. 2. Temporal evolution of the variable $x$ of two contiguous oscillators in a ring of $N=3$ Lorenz oscillators. Notice the time scale of this figure compared to that of Fig. 1. The values of the parameters are the same as in Fig. 1.

et al., 1994]). The time evolution of small differences around the synchronized state is governed by the equation,

$$
\dot{\delta \mathbf{x}}=\mathbf{H} \delta \mathbf{x}
$$

where the $\mathbf{H}$ matrix is organized in a series of blocks corresponding to the uncoupled oscillators plus a number of off-diagonal terms arising from coupling.

However, the structure of this matrix is circulant, and for this reason one can put these equations in a more convenient form through the use of Discrete Fourier Transform (DFT) [Turing, 1952; 
Heagy et al., 1994]. As a result, the following equations are obtained,

$$
\dot{\eta}^{(k)}=\mathbf{C}^{(k)} \eta^{(k)}
$$

where in the case of the Lorenz model the structure of each block can be cast in the form,

$$
\mathbf{C}^{(k)}=\left(\begin{array}{ccc}
-\sigma & \sigma & 0 \\
\left(R e_{k}-z\right) & -1 & -x \\
y & x & -b
\end{array}\right),
$$

with $e_{k}=\exp (i 2 \pi k / N)$ and being $k=0, \ldots$, $(N-1)$ the Fourier modes of the system.

The $\mathbf{C}^{(k)}$ matrices have time-dependent (chaotically varying) coefficients, and, thus, we have chosen to characterize its stability by determining the corresponding Lyapunov spectrum considering the infinite-time limit of the real part of the eigenvalues of this matrix. This has been done by generalizing Wolf's algorithm [Wolf et al., 1985] to the case of complex vector spaces, while the different possible values of $k$ and $N$ have been joined through the definition of the reduced wavenumber $q=k / N$, and this yields the function $\lambda(q)$, that represents the highest Lyapunov exponent as a function of this variable. According to linear stability theory [Heagy et al., 1994] the stability of the synchronized state will occur whenever the transverse Lyapunov exponent is negative. However, if one assumes that the dependence of $\lambda$ on $q$ is smooth, the fact that the uniform dynamics is chaotic, i.e. that $\lambda(0)>0$, implies that the uniform chaotic state must be unstable for perturbations of some characteristic wavelength (see also [Bohr et al., 1987] for an analogous argument).

In a more quantitative fashion, it can be shown that for the parameters used in this work, and reported in Fig. 3, this crossing occurs for $q_{c} \sim 0.37$, what implies that it occurs already for $N=3$. This can be confirmed through numerical simulation of Eq. (1), as can be seen in Fig. 2. An easily acknowledged point from these results is that when the instability occurs the behavior of each oscillator becomes periodic, and neighboring oscillators exhibit a phase difference of $2 \pi / N$. The first aspect, i.e. the transition from chaotic to periodic cannot be explained in the framework of a linear stability theory, and the observed behavior implies, probably, a global bifurcation.

However, the other aspect can be understood by noticing that the instability occurs through a

\section{$\lambda(q)$}

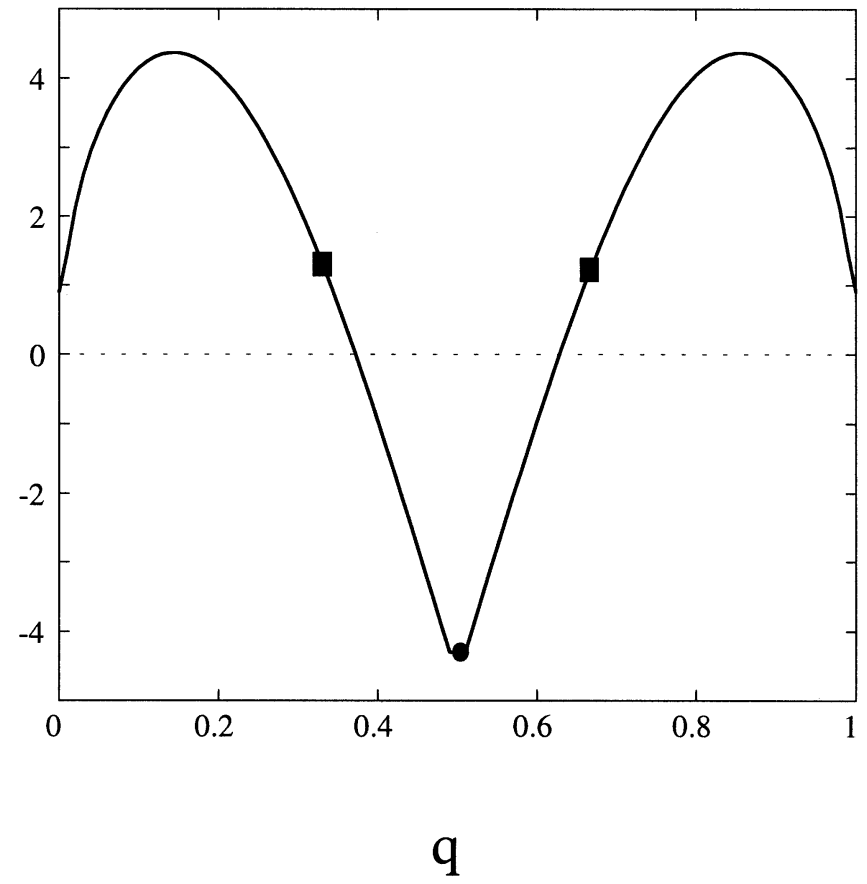

Fig. 3. Representation of the highest Lyapunov exponent $\lambda(q)$ as a function of $q=k / N$, i.e. $\lambda(q)$ versus $q$. The circles indicate the highest transverse Lyapunov exponent for a ring of $N=2$ Lorenz oscillators, whereas the squares indicate the same for $N=3$. The values of the parameters are the same as in Fig. 1.

symmetric Hopf bifurcation [Collins \& Stewart, 1994]. This bifurcation is allowed because the presence of the $e_{k}$ terms in Eq. (4) implies that half of the Fourier modes are complex conjugate to other half. In particular this implies that when a given mode crosses the instability threshold there will be another mode that also exhibits the same type of crossing. Whether these two complex conjugate unstable modes are real or complex will depend on the structure of the matrix, although in the present case, and by resorting to an approximate procedure [Güémez et al., 1997], we have shown that the modes are indeed complex. The result is immediate: A Hopf bifurcation occurs, implying the appearance of a discrete rotating wave, in which neighboring oscillators exhibit the reported phase difference.

Symmetry is here a very helpful tool as it determines the properties of the different bifurcation branches. In the case of unidirectional coupling that we are considering here, and that it is the most relevant one in the case of CPGs, symmetry indicates that a single branch of rotating waves is obtained 
[Collins \& Stewart, 1994], where a single rotation direction is allowed.

\section{Rings of Chua's Oscillators}

An analogous study to that of the previous section has been carried out with rings of Chua's oscillators, a well-known paradigm of chaos in electronic circuits. In this case the oscillators have been coupled according to the following scheme, where the evolution equations for each coupled oscillator are reported,

$$
\left.\begin{array}{l}
\dot{x}_{j}=\alpha\left[y_{j}-x_{j}-f\left(\bar{x}_{j}\right)\right] \\
\dot{y}=x_{j}-y_{j}+z_{j} \\
\dot{z}_{j}=-\beta y_{j}-\gamma z_{j}
\end{array}\right\} \quad j=1, \ldots, N .
$$

A theoretical study of this situation has been carried out in [Matías et al., 1997b], while the predictions have been confirmed experimentally in [Sánchez et al., 1997]. The nonlinear resistor $f(x)$ in (5) is given by,

$$
f(x)=\left\{b x+\frac{1}{2}(a-b)[|x+1|-|x-1|]\right\} .
$$

Driving is introduced through the nonlinear term $f(x)$ in (5), such that $\bar{x}_{k}=x_{k-1}$ for $k \neq 1$, whereas for $k=1, \bar{x}_{1}=x_{N}$.

The same type of linear stability analysis discussed in the previous section can be applied here. In this case the matrix $\mathbf{C}^{(k)}$ takes the following form,

$$
\mathbf{C}^{(k)}=\left(\begin{array}{ccc}
-\alpha\left[1+f^{\prime}(x) e_{k}\right] & \alpha & 0 \\
1 & -1 & 1 \\
0 & -\beta & -\gamma
\end{array}\right),
$$

which leads to the representation of the highest transverse Lyapunov exponent as a function of the reduced wave number, plotted in Fig. 4 . In the present case it is found that the onset of instability occurs at $q_{c}=0.21$, which implies that the ring becomes unstable when $N \geq 5$. This can be seen from Fig. 5 that presents results for $N=4$, while the behavior past the instability is presented in Fig. 6, that shows results for $N=5$. The interesting feature is now that the behavior of the oscillators is not periodic, but chaotic, while neighboring oscillators present a phase difference that is approximately equal to $2 \pi / N$. Thus, the ring can be better characterized as exhibiting a rotating chaotic wave.
Regarding the stability of chaotic synchronization as a function of the parameters of the model, by increasing $\alpha$ and $\gamma$ one sees that the critical size $N_{c}$ increases, as shown in Table 1. Thus,

\section{$\lambda(q)$}

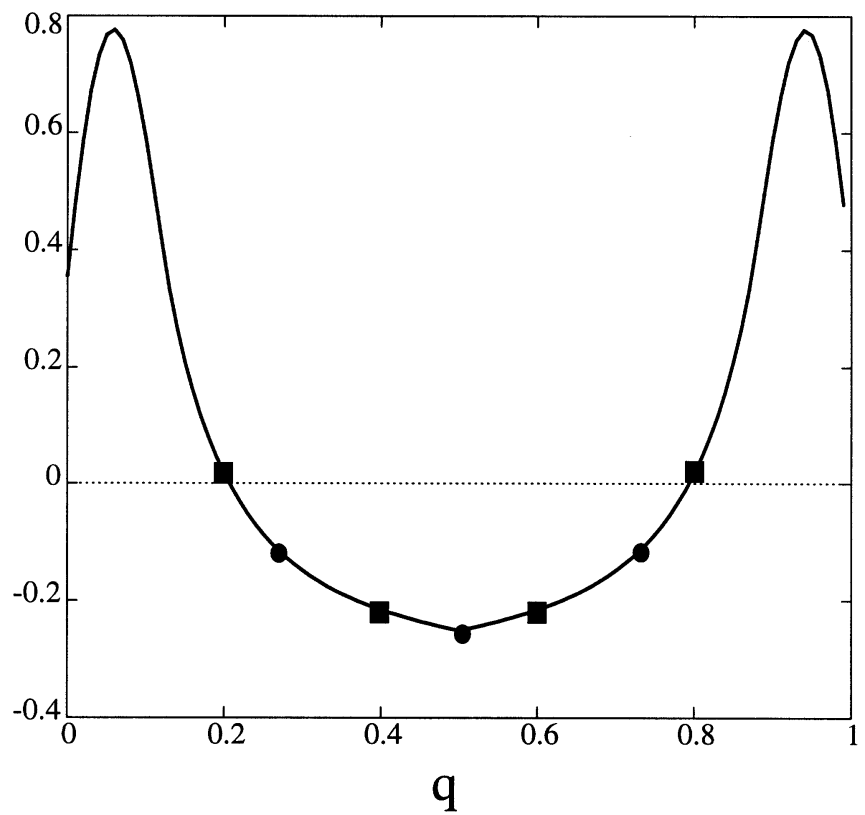

Fig. 4. Representation of the highest Lyapunov exponent $\lambda(q)$ as a function of $q=k / N$, i.e. $\lambda(q)$ versus $q$. The circles indicate the highest transverse Lyapunov exponent for a ring of $N=4$ Chua oscillators, whereas the squares indicate the same for $N=5$. The values of the parameters are $(\alpha, \beta, \gamma, a, b)=(10,14.87,0.06,-1.27,-0.68)$.

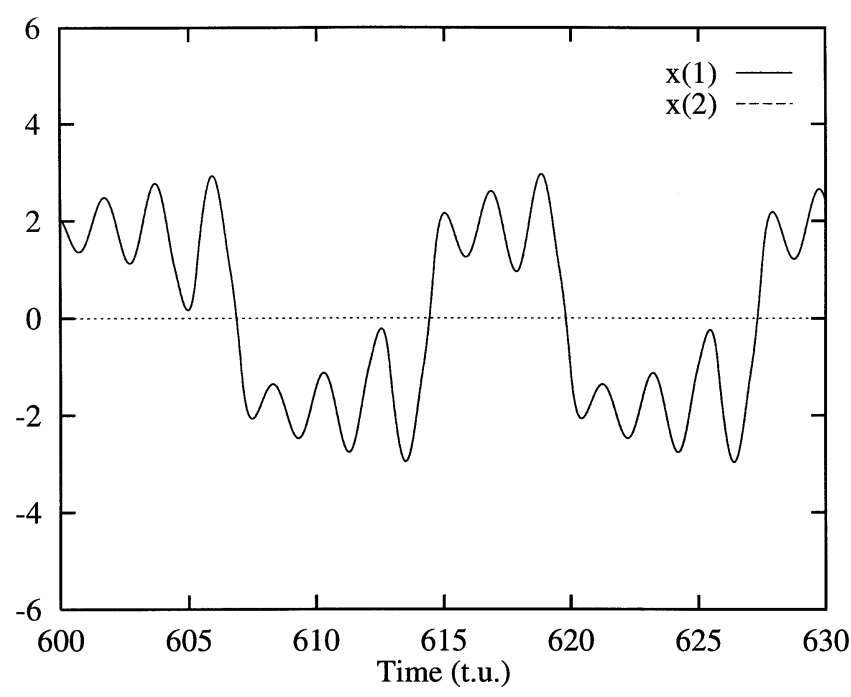

Fig. 5. Temporal evolution of the variable $x$ of two contiguous oscillators in a ring of $N=4$ Chua oscillators. The values of the parameters are the same as in Fig. 4. 


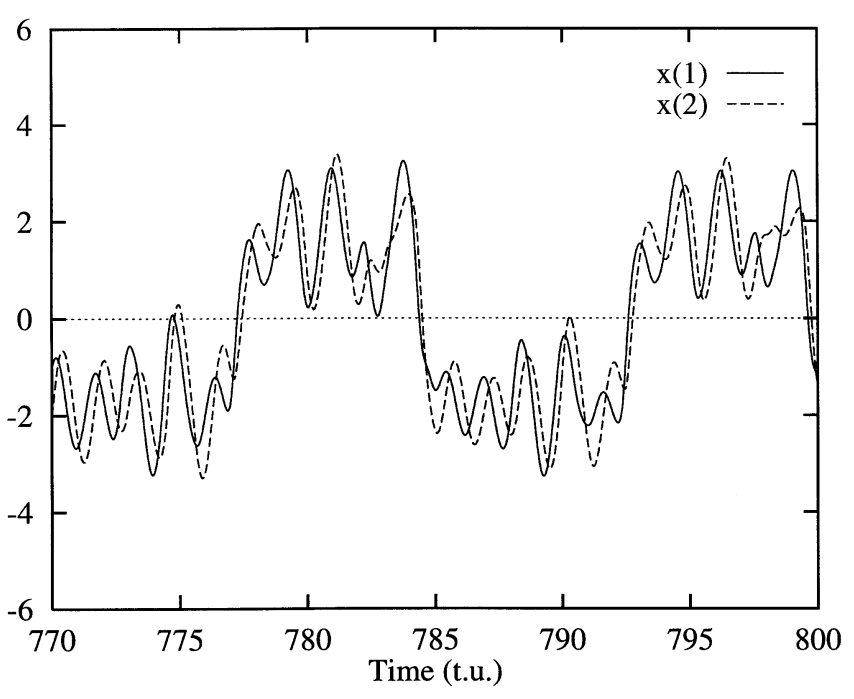

Fig. 6. Temporal evolution of the variable $x$ of two contiguous oscillators in a ring of $N=5$ Chua oscillators. The values of the parameters are the same as in Fig. 4.

Table 1. Critical number of chaotic Chua's circuits in a ring, $N_{c}$, that supports chaotic (uniform) synchronization.

\begin{tabular}{ll}
\hline$N_{c}$ & \multicolumn{1}{c}{$(\alpha ; \gamma)$} \\
\hline 4 & $\alpha=10 ; \gamma \leq 0.15$ \\
5 & $\alpha=10 ; 0.15<\gamma<0.2$ \\
6 & $\alpha=12 ; \gamma=0.2$ \\
\hline
\end{tabular}

for the value $\gamma=10$ used in all our calculations for $\gamma \leq 0.15$ one gets $N_{c}=4$, while when $0.15<\gamma<$ 0.20 this critical value becomes $N_{c}=5$. Considering higher values of $\gamma$ is meaningless, as the system becomes periodic. However, one can stabilize the $N=6$ ring in the chaotic synchronized state by increasing simultaneously $\alpha$ (e.g. for $\alpha=12$ and $\gamma=0.2$ ).

\section{Conclusions}

In the present work we have considered the behavior of rings of unidirectionally coupled identical chaotic oscillators. In particular, we have considered the cases of Lorenz and Chua systems. In both cases it is found that the uniform chaotic state of the ring is unstable to perturbations of some finite wavelength, or, equivalently, a finite size $N$. This leads to an instability in the uniform state, that is conveniently characterized by performing a Discrete Fourier Transform on the linear stability ma- trix of the problem. However, the behavior exhibited by these two types of rings is different in that in one case (Lorenz system) one obtains discrete periodic rotating waves, while in the second case (Chua system) these waves are chaotic.

These discrete spatiotemporal structures are interesting in the context of dynamical systems theory, as they reveal the richness of dynamical behaviors that one may obtain in coupled arrays of chaotic oscillators, beyond synchronization. In addition, they can be potentially useful in connection to CPGs, i.e. rings of coupled neuron models. It has been found that these structures can be useful in locomotion, where the different symmetrybreaking solutions would be responsible for the different gaits that the animal exhibits [Collins \& Stewart, 1994]. In particular, in this context it is useful to notice that in the case of rings of Lorenz systems the pattern that emerges is periodic, although the dynamics of the uncoupled oscillators was chaotic. It is also interesting to notice the time scale of the emerging rotating wave: It is, at least, one order of magnitude faster than the uncoupled oscillators, and this could be relevant in a neuronal context. As well, one should bear in mind that the brain is able to perform various tasks in a short time, although the neurons in which these tasks base are relatively slow.

\section{Acknowledgments}

We would like to thank N. Lorenzo for helpful discussions. This work was supported in part by DGICYT (Spain) Research Grants No. PB95-0570 and No. PB96-0937.

\section{References}

Bohr, T., Grinstein, G., He, Y. \& Jayaprakash, C. [1987] "Coherence, chaos, and broken symmetry in classical, many-body systems," Phys. Rev. Lett. 58, 2155-2158.

Collins, J. J. \& Stewart, I. [1994] "A group-theoretic approach to rings of coupled biological oscillators," Biol. Cybern. 71, 95-103.

Fujisaka, H. \& Yamada, T. [1983] "Stability theory of synchronized motion in coupled-oscillator systems," Prog. Theor. Phys. 69, 32-47.

Güémez, J. \& Matías, M. A. [1995] "Modified method for synchronizing and cascading chaotic systems," Phys. Rev. E52, R2145-R2148.

Güémez, J., Martín, C. \& Matías, M. A. [1997] "Approach to the chaotic synchronized state of some driving methods," Phys. Rev. E55, 124-134. 
Hayashi, H. \& Ishizuka, S. [1992] "Chaotic nature of bursting discharges in the Onchidium pacemaker neuron," J. Theor. Biol. 156, 269-291.

Heagy, J. F., Carroll, T. L. \& Pecora, L. M. [1994] "Synchronous chaos in coupled oscillator systems," Phys. Rev. E50, 1874-1885.

Matías, M. A., Pérez-Muñuzuri, V., Lorenzo, M. N., Mariño, I. P. \& Pérez-Villar, V. [1997a] "Observation of a fast rotating wave in rings of coupled chaotic oscillators," Phys. Rev. Lett. 78, 219-222.

Matías, M. A., Güémez, J., Pérez-Muñuzuri, V., Mariño, I. P., Lorenzo, M. N. \& Pérez-Villar, V. [1997b] "Size instabilities in rings of chaotic synchronized systems," Europhys. Lett. 37, 379-384.

Pecora, L. M. \& Carroll, T. L. [1990] "Synchronization in chaotic systems," Phys. Rev. Lett. 64, 821-824.

Sánchez, E., Matías, M. A. \& Pérez-Muñuzuri, V. [1998]
"Chaotic synchronization in linear arrays and rings of chaotic systems," IEEE Trans. Circuits Syst. I, in press.

Strogatz, S. H. \& Stewart, I. [1993] "Coupled oscillators and biological synchronization," Sci. Am. 269(6), 102-109.

Tang, Y. S., Mees, A. I. \& Chua, L. O. [1983] "Synchronization and chaos," IEEE Trans. Circuits Syst. I30, 620-626.

Turing, A. M. [1952] "The chemical basis of morphogenesis," Phil. Trans. R. Soc. London B237, 37-72.

Winfree, A. T. [1980] The Geometry of Biological Time (Springer, NY).

Wolf, A., Swift, J. B., Swinney, H. L. \& Vastano, J. A. [1985] "Determining Lyapunov exponents from a time series," Physica D16, 285-317. 